\title{
Is it Possible to Test Brane-World Scenarios by Observation of Quasars and Microquasars?
}

\author{
C. H. Coimbra-Araújo, R. da Rocha, \\ IFGW, Universidade Estadual de Campinas, \\ CP 6165, 13083-970, Campinas, SP, Brazil \\ and I. T. Pedron \\ Universidade Estadual do Oeste do Paraná, \\ 85960-000 Marechal Cândido Rondon, Brazil \\ (Received on 15 October, 2005)
}

\begin{abstract}
The aim of this work is to present a possible way to estimate observational electromagnetical clues in the spectrum of quasars and microquasars due to the presence of extra dimensions. Here we analyze two possible ways to detect these electromagnetic signals: by the brane corrected accretion mechanism and by production of electromagnetic KK modes. We show that for the static black hole accretion case, the brane corrections cause a extremelly small variation in the luminosity of the quasar. For the electromagnetic KK modes case, apparently the answer for the biggest quasars is positive and on the other hand, the possibility that microquasars can present constraints on extra-dimensional gravity in their spectra is less probable.
\end{abstract}

\section{INTRODUCTION: BLACK HOLES ON THE BRANE}

For many reasons, brane-world models present a rich way to explain our universe [1]. The Einstein equations in the brane can be written as the projection of Einstein equation in the high-dimensional bulk. The bulk is an Anti-de Sitter space and has warp properties symbolized by a negative cosmological constant. Assuming $Z_{2}$ symmetry, the IsraelDarmois junction conditions, and the Bianchi identities, Einstein equations are written as

$$
\begin{aligned}
& G_{\mu v}=-\frac{1}{2} \Lambda_{5} g_{\mu v} \\
& +\frac{1}{4} \kappa_{5}^{4}\left[T T_{\mu v}-T_{v}^{\alpha} T_{\mu \alpha}+\frac{1}{2} g_{\mu v}\left(T^{2}-T_{\alpha \beta}^{\alpha \beta}\right)\right]-E_{\mu v},
\end{aligned}
$$

where $T=T_{\alpha}{ }^{\alpha}$ denotes the trace of the momentum-energy tensor $T_{\mu \nu}, \Lambda_{5}$ denotes the 5-dimensional cosmological $\mathrm{AdS}_{5}$ bulk constant, and $E_{\mu \nu}$ denotes the 'electric' components of the Weyl tensor. The constant $\kappa_{5}=8 \pi G_{5}$, where $G_{5}$ denotes the 5-dimensional Newton gravitational constant, that can be related to the 4-dimensional gravitational constant $G$. A vacuum on the brane, where $T_{\mu v}=0$ outside a black hole (for a review, see e.g. [3]), implies, by Bianchi identities, that $E_{\mu \nu}^{; v}=0$. A particular manner to express the vacuum field equations in the brane is $E_{\mu v}=-R_{\mu v}$. One can use a Taylor expansion in order to probe properties of a static black hole on the brane [2]. Thus, for a static spherical metric on the brane given by

$$
g_{\mu v} d x^{\mu} d x^{v}=-F(r) d t^{2}+\frac{d r^{2}}{H(r)}+r^{2} d \Omega^{2}
$$

where $d \Omega^{2}$ denotes the spherical 3-volume element related to the geometry of the 3-brane. The metric is led to the Schwarzschild one, if $F(r)$ equals $H(r)$. The exact determination of these radial functions remains an open problem in $\mathrm{BH}$ theory on the brane $[1,3]$.

\section{ACCRETION EFFICIENCE IN SCHWARZSCHILD QUASARS WITH CORRECTIONS}

Defining $\psi(r)$ as the deviation from a Schwarzschild form for $H(r)[1,3]$

$$
H(r)=1-\frac{2 G M}{c^{2} r}+\psi(r)
$$

where $M$ is constant, and where, for a large $\mathrm{BH}$ with horizon scale $r \gg \ell, \psi(r) \approx-\frac{4 G M \ell^{2}}{3 c^{2} r^{3}}$ [1]. Now we can explore the idea that, if black holes and specially the supermassive black holes (SMBHs) present in the nucleus of galaxies and quasars, do cause deviations from the 4D general relativity, these corrections should cause a small deviation in all SMBH properties [4]. First we need to estimate the corrected Schwarzschild brane radius $R_{\text {Sbrane }}$. This is possible doing $H=0$ :

$$
R_{\text {Sbrane }}^{3}-\frac{2 G M}{c^{2}} R_{\text {Sbrane }}^{2}-\frac{4 G M \ell^{2}}{3 c^{2}}=0 .
$$

Using Cardano's formulæ it follows that

$$
R_{\text {Sbrane }}=(a+\sqrt{b})^{1 / 3}+(a-\sqrt{b})^{1 / 3}+\frac{2 G M}{3 c^{2}},
$$

where

$$
\begin{aligned}
& a=\frac{R_{S}}{3}\left(\ell^{2}+\frac{R_{S}^{2}}{9}\right), \\
& b=\frac{R_{S}^{2} \ell^{2}}{9}\left(\ell^{2}+\frac{2 R_{S}^{2}}{9}\right),
\end{aligned}
$$

where $R_{S}$ is the classical Schwarzschild radius. Now, substituting the values of $G$ and $c$ in the SI, and adopting $\ell \sim 0.1 \mathrm{~mm}$ and $M \sim 10^{9} M_{\odot}\left(\right.$ where $\left.M_{\odot} \approx 2 \times 10^{33} \mathrm{~g}\right)$ denotes solar mass, corresponding to the mass of a SMBH, it follows from eq.(4) that the correction in the Schwarzschild radius of a SMBH by brane-world effects is given by $R_{\text {Sbrane }}-R_{S} \sim 100 \mathrm{~m}$, and since the Schwarzschild radius $R_{S}$ is defined as $\frac{2 G M}{c^{2}}=$ 
$2.964444 \times 10^{12} \mathrm{~m}$, the relative error concerning the braneworld corrections in the Schwarzschild radius of a SMBH is given by $1-\frac{R_{S}}{R_{\text {Sbrane }}} \sim 10^{-10}$. These calculations shows that there exists a correction in the Schwarzschild radius of a SMBH caused by brane-world effects, although it is negligible. Then, for the static case we must look for another kind of observational mechanism.

\section{ELECTROMAGNETIC KK MODES?}

A natural manner to detect extra dimensions is by detection of gravitational waves due to extradimensional or KaluzaKlein (KK) modes. Here on we will use the method developed by [5]. Our assumption is to consider the conversion of these gravitational KK modes in eletromagnetic radiation. For this we will assume a generic black string background without charge producing a static Reissner-Nordström black hole on the brane. This background is given by

$$
\begin{aligned}
d s^{2} & =e^{-2|y| / \ell}\left[-(1-2 G M / r-f) d t^{2}\right. \\
& \left.+\frac{d r^{2}}{1-2 G M / r-f}+r^{2} d \Omega^{2}\right]+d y^{2}
\end{aligned}
$$

where $f$ is a function independent of the charge. Using the field equations found in eq. (1), it is possible to find the pertubative equations, assuming that the bulk cosmological constant is given by $\Lambda_{5}=-6 \ell^{-2}$ [1]. Here we must also assume the eletro-vacuum energy-momentum tensor. The perturbed metric is $\bar{g}_{\mu v}=g_{\mu v}+h_{\mu v}$. Then we found the follow metric perturbations equations

$$
\begin{array}{r}
k_{\mu v, \lambda}{ }^{\lambda}-k_{\mu, v, \lambda}^{\lambda}-k_{v, \mu, \lambda}^{\lambda}-\frac{1}{2} g_{\mu v} k_{, \lambda, \lambda}-\frac{6}{\ell^{2}} h_{\mu v} \\
-\frac{1}{4} \kappa_{5}^{4}\left[\left(h^{\lambda \rho} F_{\alpha \lambda} F_{\lambda}^{\alpha}-\frac{1}{2} h F_{\lambda \rho} F^{\lambda \rho}\right) T_{\mu v}\right. \\
+T_{\alpha}^{\alpha}\left(\delta F_{\mu \lambda} F_{v}{ }^{\lambda}+\delta F_{v \lambda} F_{\mu}^{\lambda}-\frac{1}{2} g_{\mu \nu} \delta F_{\lambda \rho} F^{\lambda \rho}\right.
\end{array}
$$

$$
\begin{aligned}
& \left.+h^{\lambda \rho} F_{\mu \lambda} F_{v \rho}-\frac{1}{2} g_{\mu v} h^{\lambda \rho} F_{\lambda \alpha} F_{\rho}{ }^{\alpha}-\frac{1}{4} h_{\mu C} F_{\lambda \rho} F^{\lambda \rho}\right) \\
& -\left(\delta F_{\lambda}^{C}{ }_{\lambda} F_{v}^{\lambda}+\delta F_{v \lambda} F_{C}{ }^{\lambda}-\frac{1}{2} g_{C v} \delta F_{\lambda \rho} F^{\lambda \rho}\right. \\
& \left.+h^{\lambda \rho} F_{C \lambda} F_{v \rho}-\frac{1}{2} g_{C v} h^{\lambda \rho} F_{\lambda \alpha} F_{\rho}^{\alpha}-\frac{1}{4} h_{C \nu} F_{\lambda \rho} F^{\lambda \rho}\right) T_{\rho C} \\
& -T^{C}{ }_{v}\left(\delta F_{\mu \lambda} F_{C}{ }^{\lambda}+\delta F_{C \lambda} F_{\mu}{ }^{\lambda}-\frac{1}{2} g_{\mu C} \delta F_{\lambda \rho} F^{\lambda \rho}\right. \\
& \left.+h^{\lambda \rho} F_{\mu \lambda} F_{C \rho}-\frac{1}{2} g_{\mu C} h^{\lambda \rho} F_{\lambda \alpha} F_{\rho}^{\alpha}-\frac{1}{4} h_{\mu \nu} F_{\lambda \rho} F^{\lambda \rho}\right) \\
& +\frac{1}{2} h_{\mu v}\left[(\operatorname{TrT})^{2}-\left(\operatorname{TrT}^{2}\right)\right]+\mathrm{g}_{\mu \nu} \mathrm{T}_{\alpha}{ }^{\alpha}\left(\mathrm{h}^{\lambda \rho} \mathrm{F}_{\lambda \alpha} \mathrm{F}_{\rho}{ }^{\alpha}\right. \\
& \left.-\frac{1}{2} h F_{\lambda \rho} F^{\lambda \rho}\right)-\frac{1}{2} g_{\mu v}\left(\delta F_{\alpha \lambda} F_{\beta}^{\lambda}+\delta F_{\beta \lambda} F_{\alpha}{ }^{\lambda}\right. \\
& -\frac{1}{2} g_{\mu \beta} \delta F_{\lambda \rho} F^{\lambda \rho}+h^{\lambda \rho} F_{\alpha \lambda} F_{\beta \rho}-\frac{1}{2} g_{\alpha \beta} h^{\lambda \rho} F_{\lambda \alpha} F_{\rho}{ }^{\alpha} \\
& \left.-\frac{1}{2} h_{\alpha \beta} F_{\lambda \rho} F^{\lambda \rho}\right) T^{\alpha \beta}-\frac{1}{2} g_{\mu \nu} T^{\alpha \beta}\left(\delta F_{\lambda}^{\alpha} F^{\beta \lambda}\right. \\
& +\delta F^{\beta}{ }_{\lambda} F^{\alpha \lambda}-\frac{1}{2} g^{\alpha \beta} \delta F_{\lambda \rho} F^{\lambda \rho}+h^{\lambda \rho} F_{\lambda}^{\alpha} F_{\rho}^{\beta} \\
& \left.\left.-\frac{1}{2} g^{\alpha \beta} h^{\lambda \rho} F_{\lambda \alpha} F_{\rho}^{\alpha}-\frac{1}{2} h^{\alpha \beta} F_{\lambda \rho} F^{\lambda \rho}\right)\right]-\delta E_{\mu \nu}=0,
\end{aligned}
$$

where $k_{\mu v}=h_{\mu v}-\frac{1}{2} h g_{\mu v}$. Maybe could be possible to find eletromagnetic clues indicating the 'smoke-gun' influence of KK modes in the electromagnetical spectra of quasars. Small black holes will not produce 'alongated' black strings in the extradimension, and then will fall in the 'forbidden' region showed by [5]. This discard the observational presence of electromagnetic KK modes coming from microquasars. The possibility remains for supermassive black hole/quasar case. Although Gerlach [6] argues that all eletromagnetic waves will be transformed in gravitational waves in the ReissnerNordström background, Sibgatullin [7] and Novikov \& Frolov [8] claim that perturbations in charged black holes can produce both eletromagnetic and gravitational waves. For example, in these kind of black holes, high frequency eletromagnetic wave with amplitude $A_{\text {in }}$ and impact parameter $b$, unless it is supposed to be captured, will create outgoing eletromagnetic and gravitational waves with amplitudes $A_{\text {out }}$ and $H_{\text {out }}$. If this can really happens, then it is possible to detect eletromagnetic KK modes in quasars spectra.
[1] R. Maartens. Living Rev. Relat. 7, 7 (2004) [gr-qc/0312059].

[2] N. Dadhich, R. Maartens. P. Papadopoulos, and V. Rezania. Phys. Lett. B 487, 1 (2000).

[3] P. Kanti, I. Olasagasti, and K. Tamvakis. Phys. Rev. D 68, 124001 (2003) [hep-th/0307201].

[4] C. H. Coimbra-Araújo, R. da Rocha, and I. T. Pedron. Int. J. Mod. Phys. D 14, to appear [astro-ph/0505132]; R. da Rocha and C. H. Coimbra-Araújo. [astro-ph/0509363]; [astro-ph/0510318].

[5] S. S. Seahra, C. Clarkson, and R. Maartens. Phys. Rev. Lett. 94, 121302 (2005).

[6] U. H. Gerlach. Phys. Rev. Lett. 32, 1023 (1974).

[7] N. R. Sibgatullin. Zh. Eksp. Teor. Fix. 66, 1187 (1974).

[8] I. D. Novikov, and V. P. Frolov. Physics of Black Holes, Kluwer, Dordrecht (1989). 